\title{
Pazopanib Regimen
}

National Cancer Institute

\section{Source}

National Cancer Institute. Pazopanib Regimen. NCI Thesaurus. Code C160105.

A chemotherapy regimen consisting of pazopanib that may be used in the treatment of soft tissue and uterine sarcomas; follicular, medullary, papillary, and Hurthle cell thyroid carcinomas; gastrointestinal stromal tumors (GISTs); and kidney cancer. 